\title{
On the Permeability Inside the Granular Media
}

\author{
Chen-Yuan Chen \\ Ph.D. Student, Department of Marine Environment and Engineering, National Sun Yat-Sen University, Kaohsiung, \\ Taiwan, R.O.C. \\ Chung-Pan Lee \\ Professor, Department of Marine Environment and Engineering, National Sun Yat-Sen University, Kaohsiung, Taiwan, \\ R.O.C., cplee@mail.nsysu.edu.tw \\ Cheng-Wu Chen \\ Ph.D. Candidate, Department of Civil Engineering, National Central University, Chung-Li, Taiwan, R.O.C.
}

Follow this and additional works at: https://jmstt.ntou.edu.tw/journal

Part of the Engineering Commons

\section{Recommended Citation}

Chen, Chen-Yuan; Lee, Chung-Pan; and Chen, Cheng-Wu (2002) "On the Permeability Inside the Granular Media," Journal of Marine Science and Technology. Vol. 10: Iss. 2, Article 9.

DOI: $10.51400 / 2709-6998.2312$

Available at: https://jmstt.ntou.edu.tw/journal/vol10/iss2/9

This Research Article is brought to you for free and open access by Journal of Marine Science and Technology. It has been accepted for inclusion in Journal of Marine Science and Technology by an authorized editor of Journal of Marine Science and Technology. 


\section{On the Permeability Inside the Granular Media}

\section{Acknowledgements}

This research is accomplished in hydraulic laboratory, National Sun Yat-sen University, Kaohsiung, Taiwan. This work was supported by National Science Council, Taiwan, under Grant No. NSC89-2611-E-110- 019 


\title{
ON THE PERMEABILITY INSIDE THE GRAINULAR MEDIA
}

\author{
Chen-Yuan Chen*, Chung-Pan Lee**, and Cheng-Wu Chen***
}

Key words: porous media, intrinsic permeability, dimensionless turbulent coefficient, vortex, convergence, divergence, porosity.

\begin{abstract}
The permeability and resistance characteristics in porous structures with different grain size and shape have been studied experimentally in the paper. Two materials used in the study are crushed gravels and spherical glass balls with four sizes of $5.2 \mathrm{~mm}, 7$. $4 \mathrm{~mm}, 12.2 \mathrm{~mm}$, and $31.7 \mathrm{~mm}$ for gravels and $13.0 \mathrm{~mm}, 16.0 \mathrm{~mm}, 25$. $0 \mathrm{~mm}$, and $35.0 \mathrm{~mm}$ for balls, respectively. The permeability determined from linear Darcy Law shows a strong dependence on flow velocity in addition to grain sizes and shapes. This is not consistent to the traditional definition of permeability that is only a function of material and fluid. On the other hand, if the resistance force in porous media is quantified by the sum of linear frictional drag and nonlinear form drag suggested by Ward (1964) the turbulent frictional coefficient shows a similar trend as that of Shield Curve. The authors analyze the experimental raw data via former experienced equation (Sollitt and Cross, 1972) and compare with a new equation derived according to real physical concept considering total combined drag forces. This indicates that the intrinsic permeability change significantly as the grain size Reynolds number varies and is not constant in a non-uniform flow field such as in a wave field. Results show that porous-structure characteristics like intrinsic permeability and turbulent coefficient tend to stabilize under high Reynolds number, and description with new equation is better than former equation. The characteristics of resistance force in porous media are therefore deserved further study in order to improve related applications on coastal porous structures.
\end{abstract}

\section{INTRODUCTION}

The study of the convective transport properties of fluid in porous media is a fundamental issue both theoretically and technologically. Many different models

Paper Submitted 04/10/02, Accepted 08/07/02. Author for Correspondence: Chen-Yuan Chen.

*Ph.D. Student, Department of Marine Environment and Engineering, National Sun Yat-Sen University, Kaohsiung, Taiwan, R.O.C.

**Professor, Department of Marine Environment and Engineering, National Sun Yat-Sen University, Kaohsiung, Taiwan, R.O.C. E-mail: cplee@mail.nsysu.edu.tw

***Ph.D. Candidate, Department of Civil Engineering, National Central University, Chung-Li, Taiwan, R.O.C. have been developed to define a suitable set of geometrical parameters able to describe the complex interplay between geometry, connectivity and transport properties. Fluid flow in porous media takes place within complicated boundaries that makes the rigorous solution of the Navier-Stokes equations practically impossible. Frisch et al. (1986) introduced a cellular automaton fluid model to solve the Navier-Stokes equations, numerically. When the flow through a porous medium is in the regime of low Reynolds number Darcy's Law is often applicable (Dullien, 1979). This law is given by $Q=-(K / \mu) \Delta P / \Delta L$. This linear relationship, between the volumetric flow rate per unit area $Q$ and the applied pressure $\Delta P$ divided by the length $\Delta L$ of the sample, is used to define the permeability $K$ of the medium, provided that the dynamic viscosity $\mu$ of the fluid is known. A property of porous systems frequently considered is the porosity $\varepsilon$ that is the volume fraction of void space. Some effort has been made to elucidate the nature of the exponent for the power law that relates the permeability to the porosity, in some simple porous systems (McCauley, 1992; Gueguen and Palciauskas, 1994; McCarthy, 1994).

Wave reflection and transmission through a porous structure has been studied widely, especially after the theoretical study on a crib-type breakwater by Sollitt and Cross (1972). Seawalls with porous toe, caissons on rubble foundation, rubble-mound breakwaters, and submerged porous breakwaters are some types of typical porous structures. Porous structures protect lee-side wave attack by reflecting and dissipating wave energy through the viscosity-induced resistance in the porous media. Submerged breakwaters in addition may trigger the early breaking of incident waves and dissipate most of the energy. Because of the submergence of the breakwater, its application on protecting coastal area may attract more attention for environmental concern. The reflected waves and the dissipated wave energy are strongly affected by water depth, wave properties such as period and height, and structure properties. The major structure properties are porosity, size distribution and shape of the components of the porous media, and 
geometry such as the clearance of the submerged breakwater.

Owing to the importance of characteristic of permeability, researches about characteristics including grain-size, shape, surface, and porosity are demanded immediate attention. Unfortunately, at the present time, only very simplified materials properties can be used except the results of Ward (1964), Dinoy (1971), and Sollitt and Cross (1972). According to the previous results, it is shown that the permeability scales directly proportional to the square of the length ratio, and the turbulent friction coefficients are the same in similar materials. The parameter of intrinsic permeability plays a crucial role in many of the calculations and formula of the hydraulic engineering field that deal with fluid flow through porous media. Although this permeability factor has long been accepted as a constant for any particular media, (Cherry and Freeze, 1979) experiments have shown that it does vary and is not constant as was previously assumed.

In the present day, studies on wave damping characteristics of porous media under wave action are mostly placed a curb upon simple form except assumption of linear wave. Besides, we abide by the assumption of Morison equation on porous media for solving resistance force. The Morison equation (Ward, 1964) assumes that fluid forces result from drag forces and inertial forces. Drag forces due to a relatively velocity between the body and fluid, net shear and pressure forces are imposed on the body. Inertial forces due to the relatively acceleration between the body and fluid, a fluid mass is displaced and acceleration around the body, causing a local pressure gradient which applies a net load on the body. Practically, restriction on linear wave and media shape however can be overcome with numerical analysis/methods, but assumptions about resistance force in porous media were not proved appropriately. Therefore, it is caused difference between theoretical solution and experiment at present. The main reason is that the related function of resistance force, wave filed, media characteristics including porosity, permeability, shape, size et cetera aren't determined yet by theoretical and experimental foundation. For example, permeability with regard to various porous structures is still determined by Dinoy's conclusion (1971), but this is inaccurate. This inaccuracy probably reaches one/two order according to Dinoy's conclusion (Ker and Lee, 2002). For this reason, we no more than have trend outcomes via theoretical conclusion of wave damping characteristics, but have no accurate outcomes.

The key of wave damping characteristics is how the fluid flows through porous materials. Porous media characteristics were determine (Ker, 1997) via grain- size, porosity, shape, surface and so on. At the same time, characteristics above are related to resistance force in pore fluid, and characteristics of resistance force are changed with different grain media. Consequently, the ways to determine the characteristics of permeability and resistance force in proportion to different pore media is very important. The correctness of wave damping characteristics is influenced by characteristics of permeability and resistance force. The result shows that porosity and permeability proportion to miscellaneous materials. At the same time, study adopts Ward's function (1964) of resistance force and accedes new equation derived by real physical concept. We further compare the results of grain regular glass balls with irregular crushed rocks/gravels characteristics via the two methods.

\section{MOMENTUM EQUATIONS}

\section{Former Equation of Motion}

A permeable structure is considered to be anisotropic but homogeneous. The equation of motion can be written as (Sollitt and Cross, 1972)

$$
\begin{aligned}
\frac{d u_{j}}{d t} & =-\frac{1}{\rho} \frac{\partial}{\partial x_{j}}(P+\rho g z) \\
& +(\text { Resistance forces/unit mass) } \\
& \text { in the } x_{j} \text { direction }
\end{aligned}
$$

where $j=1,2$ in a two dimension flow field, $\rho$ is the water density, $\mathrm{g}$ is gravitational acceleration, $x_{1}=x, x_{2}$ $=z$ where the origin of the coordinate system is at the still water level, $u_{1}=u, u_{2}=w$, and $P$ is the pressure. In general, the resistance forces include (1) skin friction drag in laminar flow and form drag in turbulent flow and (2) the virtual force that is due to the relative acceleration between the flow and an obstacle in the flow. The resistance forces vanish outside porous structure where an inviscid fluid is assumed.

Empirical, skin friction is proportional to $u_{j}$, and the form drag is proportional to $u_{j}\left|u_{j}\right|$. The equation was established by Ward (1964) for large porous media in steady flows. The virtual force is proportional to $d u_{j} /$ $d t$. Thus, Eq. (1) can be rewritten as

$$
\frac{d u_{j}}{d t}=-\frac{1}{\rho} \frac{\partial}{\partial x_{j}}(P+\rho g z)-\beta_{1} u_{j}-\beta_{2} u_{j}^{2}-\beta_{3} \frac{d u_{j}}{d t}
$$

where $\beta_{k j}, k=1,2,3$, are the proportional constants which can be determined empirically. The form drag and the virtual force are similar to those in the Morison equation (Steimer and Sollitt, 1978). The viscous drag forces dissipate energy. On the other hand, the virtual 
force (also called inertia drag) can be considered to contribute to the kinetic energy of an added mass that does not dissipate energy.

\section{New Drag Forces Deriving}

Consider a flow field within a multi-layer anisotropic but homogeneous porous media as shown in Figure 1(a).

Fluid flows through pores as shown in Figure 1(b) will converge and diverge before and after passes the single grain and signify the frictional drag-dominated or form drag-dominated resistance force, respectively. The corresponding frictional drag is in the form as given by Newton's viscosity law as $\tau=\mu \cdot d v / d s$. The bulk volume of a porous structure is the sum of the volume of grains and pores. The solid volume is the volume of grains, so, Bulk Volume $U_{b}=$ Solid Volume $U_{v}+$ Volume of Pores, and the number of grains = Solid Volume $U_{v} \div$ volume of each grain (Particle global volume), where

Solid Volume $U_{v}=$ cross sectional area of the meterial sample $A \times$ length of the pore media $L$ through which flow occurs. Particle global volume $=$ spherical volume $V_{s}=\pi D^{3} / 6$. The surface force can then be expressed as Surface force $=($ Dimensionless sheer coefficient $\left.C_{\tau}\right) \times($ Sheer stress $) \times($ Particle number $) \times$ (Sphere volume)

$$
\begin{aligned}
F_{\tau} & =C \tau\left(\mu \frac{u_{j}}{R_{H}}\right) \times\left(\frac{L A(1-\varepsilon)}{\pi D^{3} / 6}\right) \times\left(\pi D^{2}\right) \\
& =6 \mu \frac{C \tau(1-\varepsilon) u_{j}}{R_{H} D}
\end{aligned}
$$

where $R_{H}$ is hydraulic radius of single grain. $D$ is particle diameter, $\varepsilon$ is porosity of porous structure. $L$ is length of the pore media through which flow occurs.

In addition, form drag is associated with the pressure difference of the frontal area and the lee side of a grain. It can be estimated from the Bernoulli equation to obtain $\Delta p=\rho u 2 / 2$. The bulk drag force is calculated by integrating pressure over the area of the body.
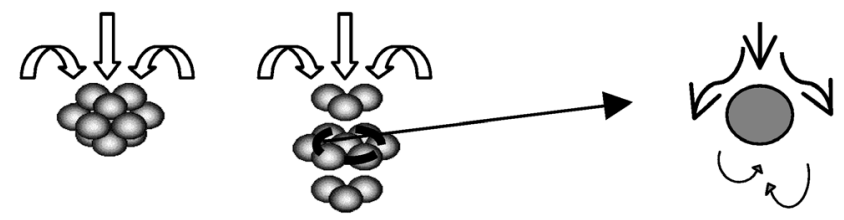

(a)

(b)

Fig. 1. (a) A schematic diagram for the flow through porous structure, (b) Convergent and Divergent flow.

$$
\begin{aligned}
& \text { Force }=\int_{\text {Area }} \operatorname{Pr} \text { essure } \cdot d(\text { Area }) \\
& \propto(\text { stagnation pressure }) \times(\text { frontal area }) \propto \rho A_{0} u^{2} / 2
\end{aligned}
$$

Let $C_{D}$ equal to the factor of proportionality, then leads to $F_{D}=C_{D} \rho A u^{2} / 2$, where $C_{D}$ is a dimensionless drag coefficient, which is function of body shape and turbulent state (Reynolds number). $\rho$ is fluid density. $A_{0}$ is frontal area, perpendicular to $u$. This results in

$$
\begin{aligned}
F_{D} & =\left(C_{D} \rho u|u| / 2\right) \times\left(\frac{L A(1-\varepsilon)}{\pi D^{3} / 6}\right) \times \pi D^{2} / 4 \\
& =\frac{3}{4} \frac{\rho C_{D}(1-\varepsilon) u|u|}{D} L A
\end{aligned}
$$

\section{EXPERIMENTAL ASSIGNMENT}

Two materials are used in the study. They are spherical glass balls and crushed gravels as shown in Figs. $2 \mathrm{a}$ and $2 \mathrm{~b}$ with four sizes of $5.2 \mathrm{~mm}, 7.4 \mathrm{~mm}, 12$. $2 \mathrm{~mm}$, and $31.7 \mathrm{~mm}$ for gravels and $13.0 \mathrm{~mm}, 16.0 \mathrm{~mm}$, $25.0 \mathrm{~mm}$, and $35.0 \mathrm{~mm}$ for balls, respectively. The corresponding porosity and intrinsic permeability are determined as following.

\section{Porosity}

Porosity, or volumetric porosity $\varepsilon$ is defined as ratio of volume of the void space $\left(U_{v}\right)$ to the bulk volume $\left(U_{b}\right)$ of a porous medium:

$$
\varepsilon=U_{v} / U_{b}=\left(U_{b}-U_{s}\right) / U_{b}
$$

Where $U_{s}$ is the volume of solids within $U_{b}$. The porosity of spherical balls used in this study is in between 0.4 to 0.41 and that of crushed rocks are 0.39 .

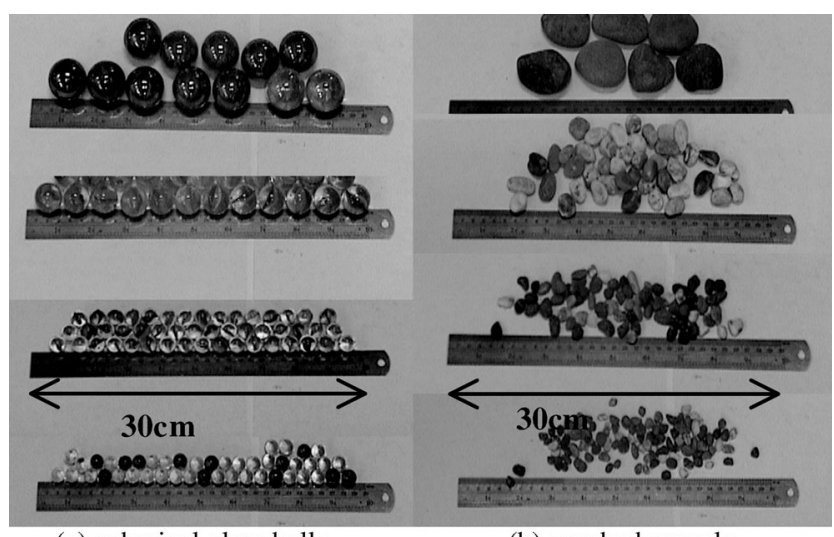

(a) spherical glass balls

Fig. 2. Photograph of eight series. 


\section{Permeability}

Darcy called this proportionality constant $K$, the permeability of the medium. $K$ depends on the grain size, shape, packing and orientation of the material in the sand tube. Permeability coefficient is the rate of flow of a fluid through a cross section of a porous mass under a unit hydraulic gradient, and permeability is the ability of a material to transmit fluid through its pores when subjected to a difference in head. A device as shown in Figures $3 a$ and $3 b$ is used to determined the permeability in the study according to formula considered in laminar flow (Darcy, 1856) as

$$
K=q d L / A d h
$$

where $K$ is permeability or called hydraulic conductivity, and $d L / d h$ is hydraulic gradient. Figure 3 shows a constant head permeability device of perspective drawing and photo. The test section is the rectangular cross-sections tank, which consists of different porous structure which is arranged by close-packed structure. Porosity and permeability are related, but they are not the same thing. A material can be very porous and hold a large volume of water, but not permit to the movement of water.

\section{Intrinsic Permeability}

Intrinsic Permeability is the ability of a porous medium to transmit fluid, and representative of the properties of the porous media alone $k=C d^{2}$, where $k$ is intrinsic permeability, $C$ is shape factor, and $d$ is particle diameter.

\section{DATA ANALYSIS}

For a steady, non-convective, simply z-directional flow in large grain permeable media, equation (2) leads to (Ward, 1964; Dinoy, 1971; Sollitt and Cross, 1972)

$$
\frac{1}{\rho} \frac{\partial}{\partial z}(\rho g z)=\frac{v \varepsilon}{k} w+\frac{C_{f} \varepsilon^{2}}{k^{1 / 2}} w^{2}
$$

Where $\rho$ is the flow density, $v$ is the kinematics viscosity, $w$ is the $\mathrm{z}$-directional fluid velocity, $C_{f}$ is the dimensionless turbulent coefficient, $\beta_{1}$ is $v \varepsilon / k$, and $\beta_{2}$ is $C_{f} e^{2} / k^{1 / 2}$. By means of equation (7), intrinsic permeability k from given constant $C_{f}$ can be calculated.

According to Hagen-Poiseuille equation $k=C_{s} R_{H}$, $R_{H}$ meant hydraulic radius is being substituted for $\varepsilon D /$ 6 as $R_{H}=$ cross section available for flow $\div$ wetted perimeter $=$ volume available for flow $\div$ total wetted surface $=\frac{\text { volume of voids / total volume }}{\text { wetted surface / total volume }}=\varepsilon D / 6$
Substituting known intrinsic permeability for equation (7), and then we get the equation as follows

$$
\frac{1}{\rho} \frac{\partial}{\partial z}(\rho g z)=\frac{v}{D^{2} \varepsilon^{2} C} w+\frac{C_{f} \varepsilon^{1 / 2}}{C^{1 / 2} D} w^{2}
$$

The new equation, also considering steady, nonconvective, simply $z$-directional flow in porous media, on the other hand leads to

$$
\frac{\gamma \Delta h}{L}=\mu \frac{\varepsilon^{1 / 2} C_{S}^{1 / 2} C_{\tau}(1-\varepsilon)}{k^{1 / 2} D} w+\frac{3}{4} \frac{\rho C_{D}(1-\varepsilon)}{D} w^{2}
$$

And

$$
\frac{\gamma \Delta h}{L}=36 \mu \frac{C_{\tau}(1-\varepsilon)}{\varepsilon D^{2}} w+\frac{3}{4} \frac{\rho C_{D}(1-\varepsilon)}{D} w^{2}
$$

\section{Shear flow analysis}

Differences of head increase in proportion to flow rate, and smaller grain-size particle has higher difference of head in constant flow condition. Considering frictional drag-dominated system, $k$ can be determined by equation (8) and is called shear flow analysis. Permeability evolution data from a series of experimental conducted at a) a constant normal atmospheric temperature ranging from $24-26^{\circ} \mathrm{C}$ and b) a uniform pore pressure assumption effected upon material across area. Results show that intrinsic permeability decreases exponentially with respect to the increase on Reynolds number regardless of materials or size. In the eight series of experiments, significant reductions in intrinsic permeability are observed, and $k$ reduction is approximately 25 percent at $13.0 \mathrm{~mm}$ diameter and more than an order of magnitude at $35.0 \mathrm{~mm}$ diameter for glass balls. Glass balls have unsteady starting compared with crushed gravels, but changes followed a near exponential form with Reynolds number constant that caused $k$ value to flatten off in less than $0.0015 \mathrm{~cm}^{2}$. For the cases of $13.0 \mathrm{~mm}$ glass balls and $12.2 \mathrm{~mm}$ crushed gravels, the

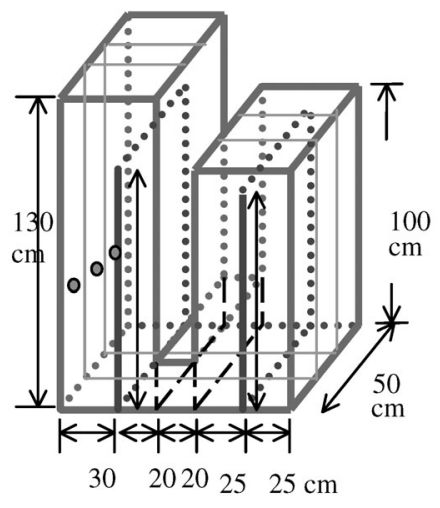

(a) 3-D perspective drawing $(\mathrm{cm})$

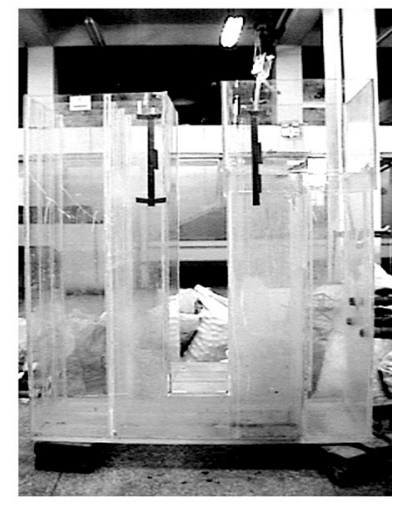

(b) Photograph
Fig. 3. Permeable instrument 
result shows they have the same trend of magnitude and slope.

\section{Vortex analysis}

According to equation (7), both frictional drag and form drag (turbulent term) have been considered simultaneously in the so-called vortex analysis. Fig. 4 shows that intrinsic permeability of $13.0 \mathrm{~mm}$ and $16.0 \mathrm{~mm}$ is constant, and $25.0 \mathrm{~mm}$ seems to be critical curve in glossy spherical glass balls. Basically, the curves have constant trend dependence on $\mathrm{R}$ in all tests. Comparing the curves of $13.0 \mathrm{~mm}$ glass balls (Fig. 4) with that of $12.2 \mathrm{~mm}$ crushed gravels (Fig. 5) shows a similar trend. Thus, grain-size dominates $k$ alone, and effect has been excluded in Figs. 6 7. However, the relationship between intrinsic permeability and grainsize is still unobvious in former equation analysis.

\section{Turbulent coefficient analysis}

Turbulent coefficient $C_{f}$ is still unknown in equation (8) for a given constant $k$. The purpose to observe $C_{f}$ dependence on Reynolds number is to grasp other characteristic elements by ignoring grain-size effect. Results show that all curves seem to have an identical constant in Fig. 6 and Fig. 7. A semi-logarithm curve has been further plotted to relate $C_{f}$ with $R$. It is found that the relationship is $\log$-linear that causes $C_{f}$ value to flatten off in range from 0.4-1.0. The trend of the curves also indicates that $C_{f}$ value is a constant and is almost independent on any materials characteristics studded here.

\section{New equation analysis}

According to equation (9), the dependence of the intrinsic permeability on Reynolds number is shown in Figures 8 and 9. The results indicate that intrinsic permeability is constant with respect to $R$ and scales directly proportional to the third power of the length ratio. In Figures 10 and 11, such power law correlations between $C_{D}$ and $R$ are well documented from former studies (Chakrabarti, 1987) in which $C_{D}$ became constant for large $R$. As for $C_{D}$ (based on cross-sectional area), it first decreases as $\mathrm{R}$ increases, and over a large range of Reynolds number, it is nearly constant. Figures 10 and 11 refer to two different surface conditions. Roughness effect is not seen in this case because of the limitation of $R$ in the study. Thus, results show drag coefficient is constant for large $R$ and is not related to other material characteristics, and intrinsic permeability is proportional to the third power of the length ratio alone.
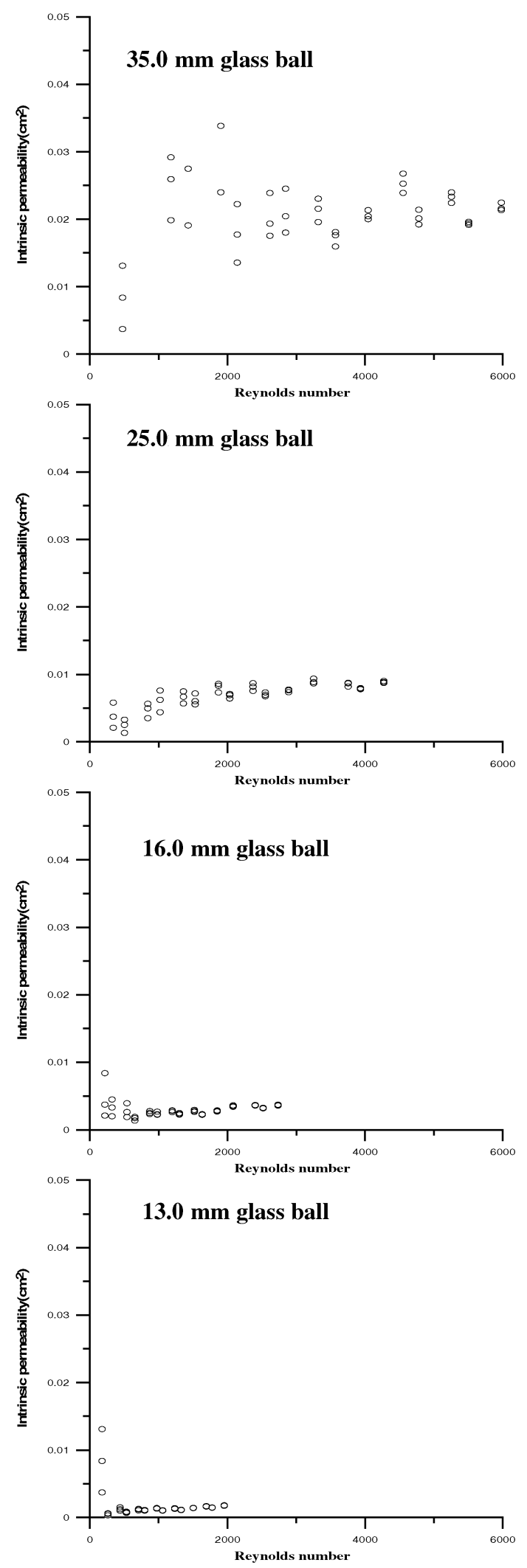

Fig. 4. Experimental raw data for intrinsic permeability dependence on Reynolds number for four series spherical glass balls, according to equation (7). 

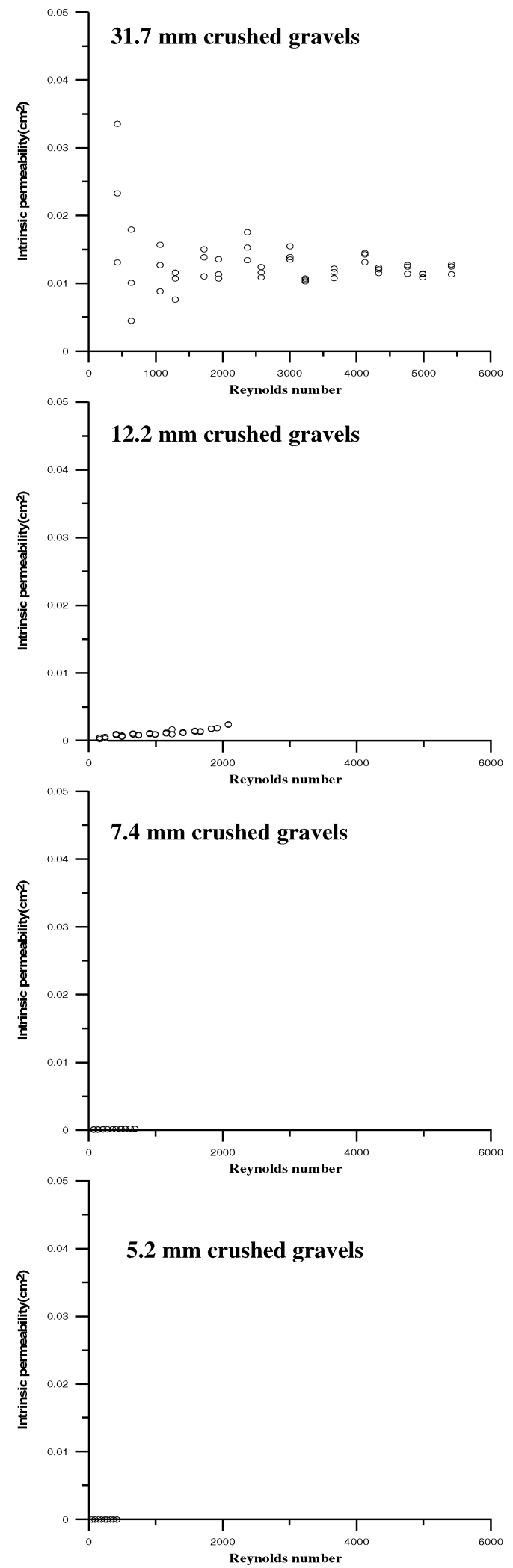

Fig. 5. Experimental raw data for intrinsic permeability dependence on Reynolds number for four series crushed gravels, according to equation (7).
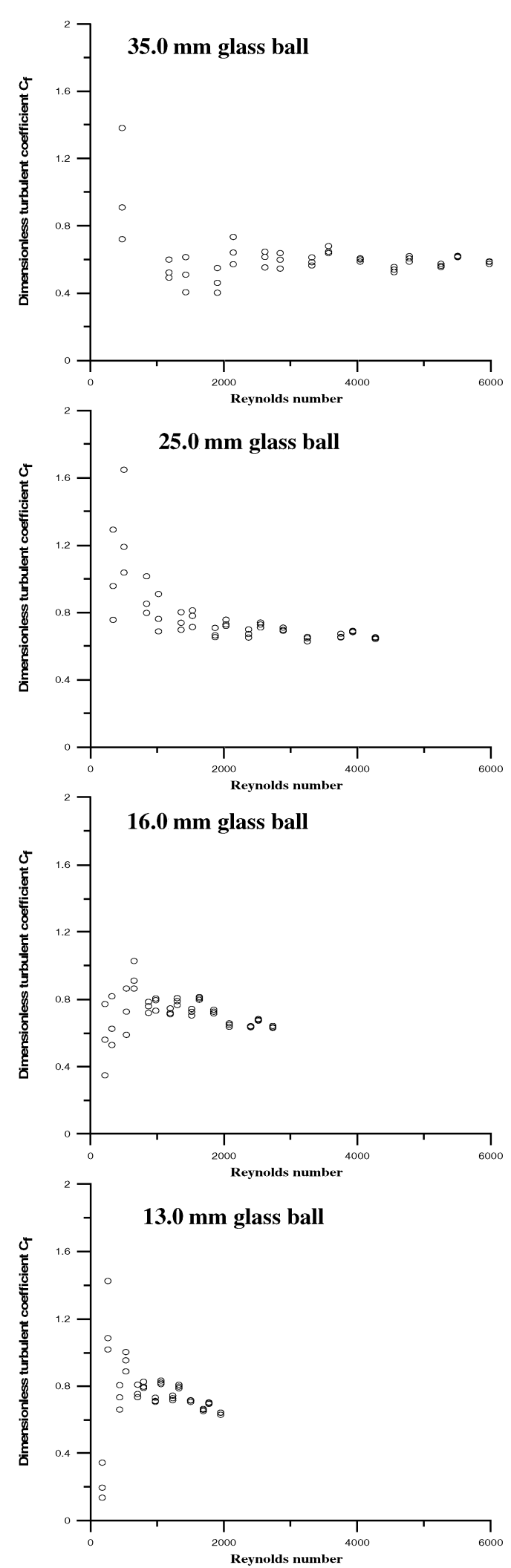

Fig. 6. Experimental raw data for turbulent coefficient dependence on Reynolds number for four series spherical glass balls, according to equation (8). 

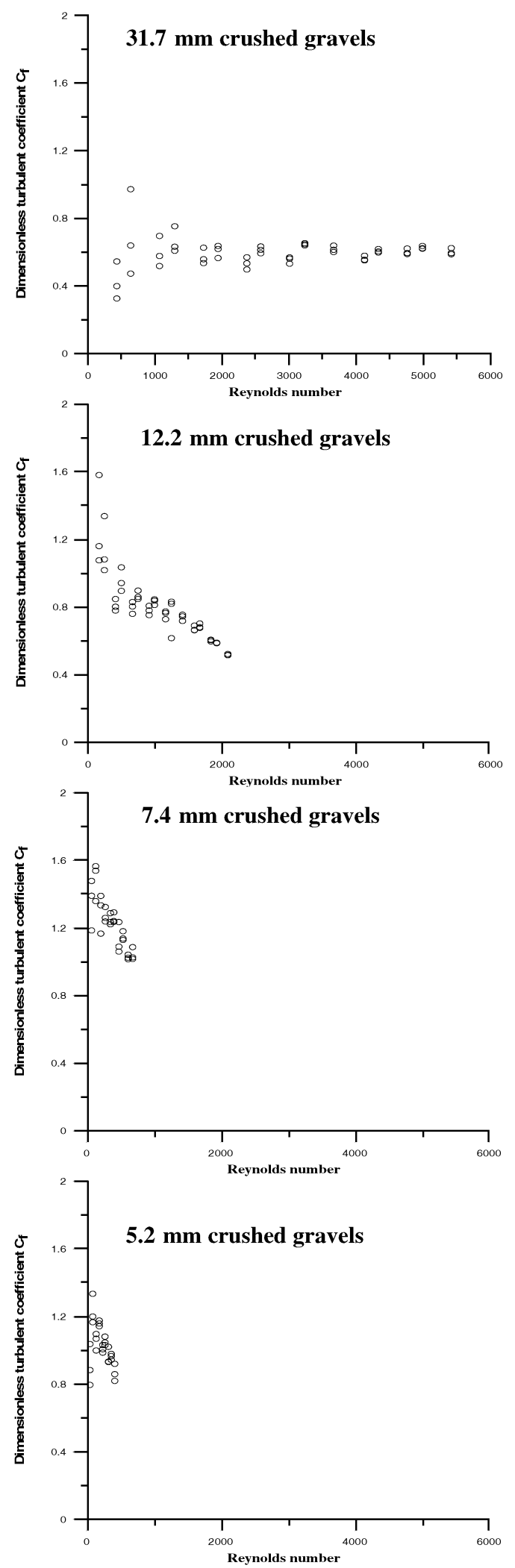

Fig. 7. Experimental raw data for turbulent coefficient dependence on Reynolds number for four series crushed gravels, according to equation (8).
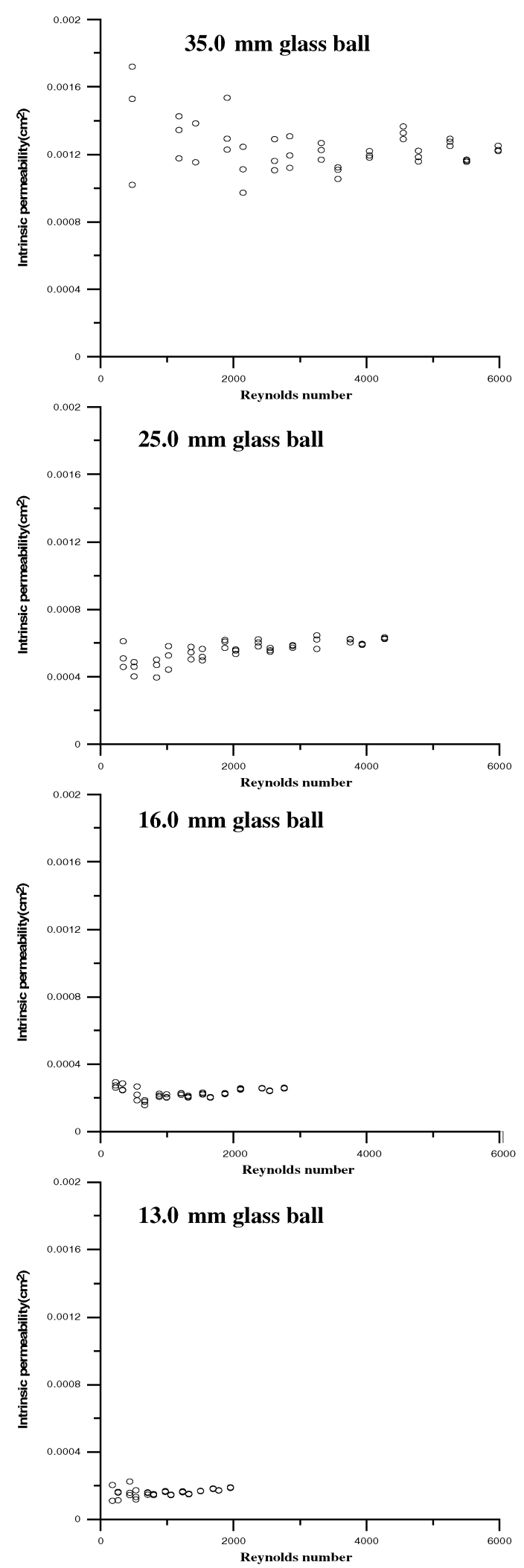

Fig. 8. Experimental raw data for intrinsic permeability dependence on Reynolds number for four series spherical glass balls, according to equation (9). 

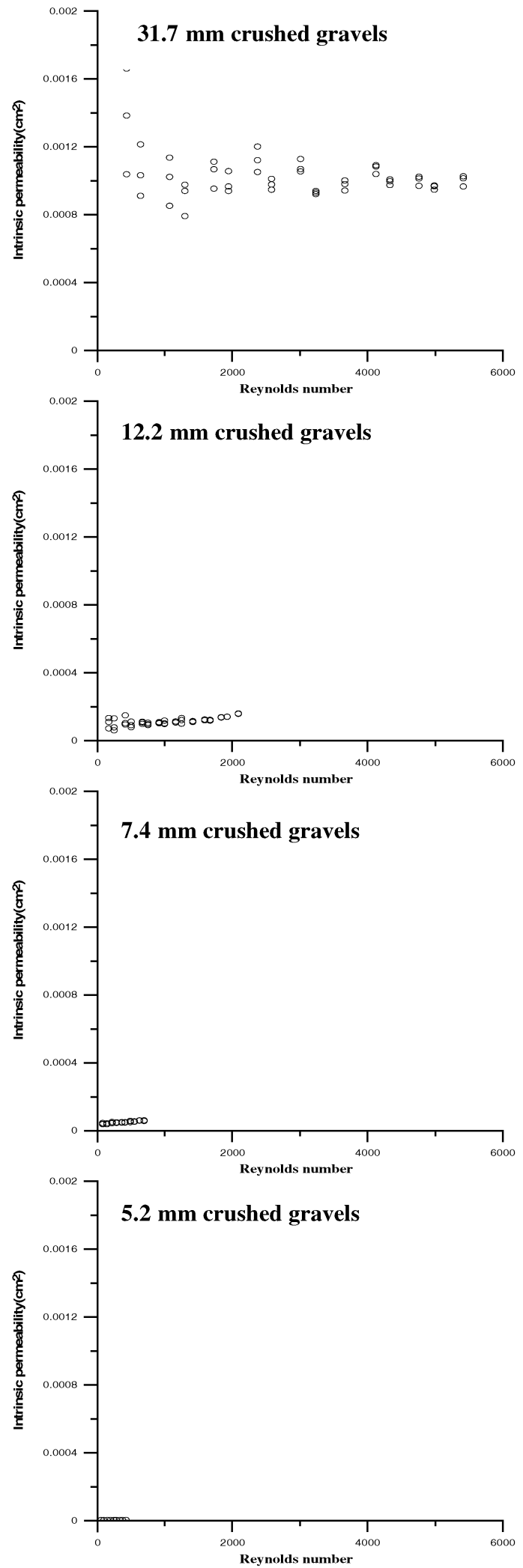

Fig. 9. Experimental raw data for intrinsic permeability dependence on Reynolds number for four series crushed gravels, according to equation (9)
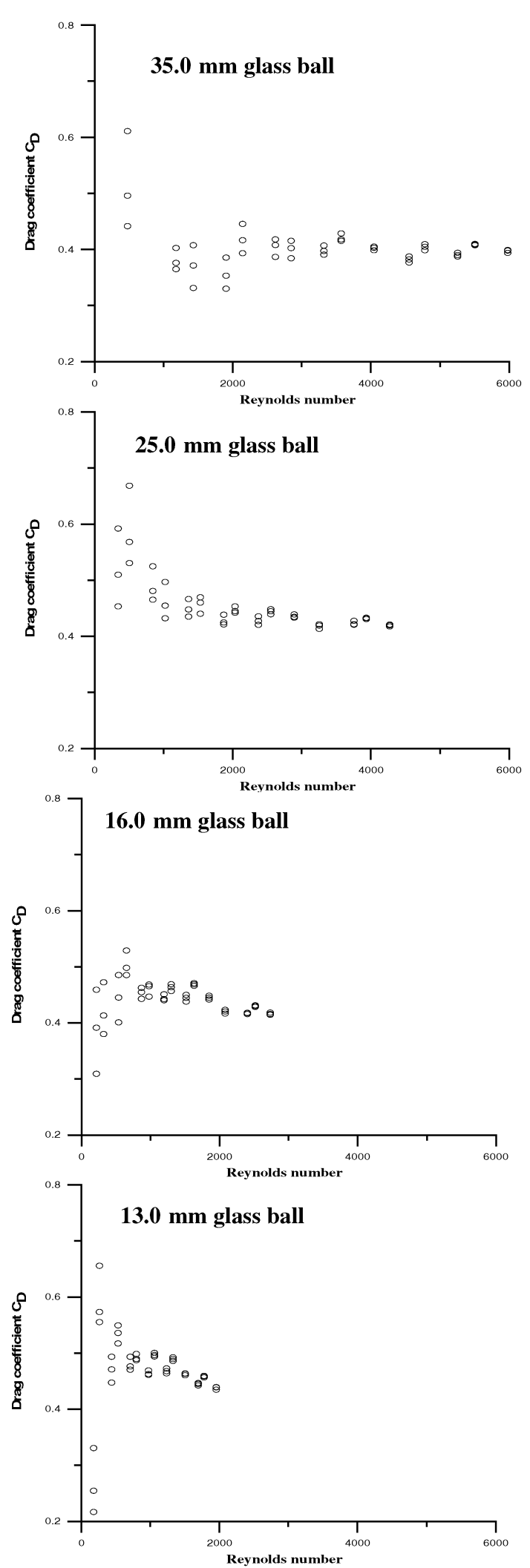

Fig. 10. Experimental raw data for drag coefficient dependence on Reynolds number for four series spherical glass balls, according to equation (10). 

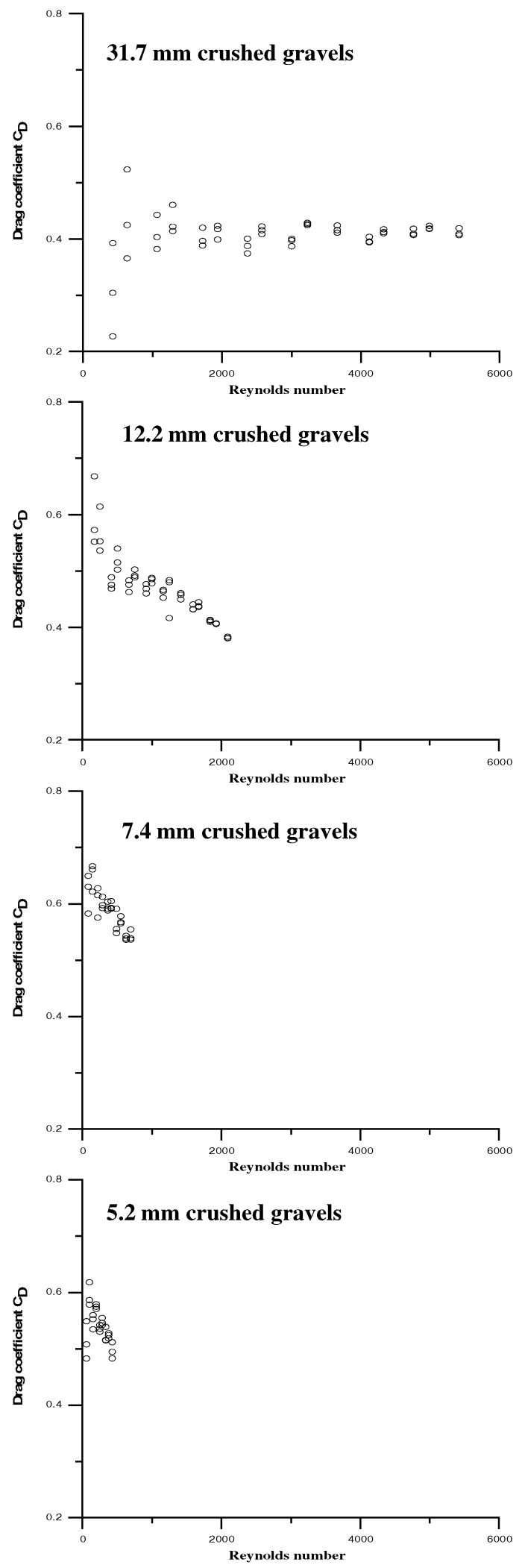

Fig. 11. Experimental raw data for drag coefficient dependence on Reynolds number for four series crushed gravels, according to equation (10).

\section{CONCLUSION}

In the study it is found that dimensionless turbulent coefficient is constant, turbulent transition occurs earlier, and so does the drag crisis when the surface is rough. Additionally, an intrinsic permeability is directly proportional to the square of the length ratio. A new equation of resistance drag in porous media has been derived from real physical concept base on convergent flow and divergent flow in pore space, and it agrees with former equation on linear frictional drag and nonlinear form drag for the test conditions. Only porosity dependence on equation needs to be determined in future experiments. In conclusion, to quicken Reynolds number would be breakthrough in next work.

\section{ACKNOWLEDGEMENT}

This research is accomplished in hydraulic laboratory, National Sun Yat-sen University, Kaohsiung, Taiwan. This work was supported by National Science Council, Taiwan, under Grant No. NSC89-2611-E-110019

\section{REFERENCE}

1. Cherry, J.A. and Freeze, R.A., Groundwater, Prentice Hall, Inc. Englewood Cliffs, New Jersey (1979).

2. Chakrabarti, S.K., "Hydrodynamics of Offshore Structures," Computational Mechanics Publications, Southampton Boston (1987).

3. Dalrymple, R.A., Losada, M.A., and Martin, P.A., "Reflection and Transmission from Porous Structures under Oblique Wave Attack," J. Fluid Mech., Vo1. 224, pp. 625-644 (1991).

4. Darcy, H., "Les Fontaines Publiques de la Ville de Dijon," Delmont, Paris (1856).

5. Dinoy, A.A., "Friction Factor and Reynolds Number Relationship in Flow through Porous Media," ME Thesis, AIT, Bangkok, Thailand (1971).

6. Dullien, F.A.L., "Porous Media," Academic Press, NY (1979).

7. Frisch, U., Hasslacher, B., and Pomeau, Y., "Lattice-Gas Automata for the Navier-Stokes Equation," Phys. Rev. Lett. Vol. 56, No. 14, 1505-1508 (1986).

8. Gueguen, Y. and Palciauskas, V., "Introduction to the Physics of Rocks," Princeton University Press, Princeton (1994)

9. Ker, W.K., "Wave Interaction with porous Tension Leg Platform," Master Thesis, Department of Marine Environment and Engineering, National Sun Yat-Sen University, Kaohsiung, Taiwan (1997).

10. Ker, W.K. and Lee, C.P., "Interaction of Waves and a Porous Tension Leg Platform," Journal of Waterway, 
Port, Coastal, and Ocean Engineering, Vol. 128, Issue 2, pp. 88-95, ASCE (2002).

11. Lee, C.P., "Wave Interaction with Permeable Structures," Ph.D. dissertation, Civil Engineering Department, Oregon State University, Corvallis, Oregon, USA (1987).

12. Madsen, O.S., "Wave Transmission Through Porous Structures," J. Waterway, Harbour and Coastal Eng. Div., ASCE, Vol. 100, No. 3, pp. 169-188 (1974).

13. Madsen, O.S. and White, S.M., "Wave Transmission Through Trapezoidal Breakwaters," Proc. of ICCE, ASCE (1976).

14. McCarthy, J.F., Phys. Fluids, p. 435 (1994).

15. McCarthy, J.F., "Flow Through Arrays of Cylinders: Lattice Gas Cellular Automata Simulations." Phys. Fluids, Vol. 6, No. 2, pp. 435-437.

16. Rojanakamthorn, S., Isobe, M., and Watanabe, A., “A
Mathematical Model of Wave Transmission Over a Submerged Breakwater," Coastal Engineering in Japan, Vol. 32, No. 2, pp. 209-233 (1989).

17. Sollitt, C.K. and Cross, R.H., "Wave transmission through permeable breakwater," Proceeding of 13th ICCE, ASCE, pp. 1827-1846 (1972).

18. Steimer, R.B., MS Thesis, Department of Civil Engineering, Oregon State University, USA (1977).

19. Steimer, R.B. and Sollitt, C.K., "Non-Conservative Wave Interaction with Fixed Semi-Immersed Rectangular Structure," $16^{\text {th }}$ ICCE, ASCE, pp. 22209-2227 (1978).

20. Sulisz, W., "Wave Reflection and Transmission Through Permeable Breakwaters," Proc. $13^{\text {th }}$ ICCE, Vol. III, pp. 1827-1846 (1985).

21. Ward, J.C., "Turbulent flow in porous media," J. of the Hydraulics Division, ASCE (1964). 